\title{
A high-current hollow cathode as a source of intense line radiation in the vuv
}

\author{
K Danzmann, J Fischer and M Kühne \\ Physikalisch-Technische Bundesanstalt, Institut Berlin, Abbestr. 2-12, D-1000 Berlin 10. \\ Germany
}

Received 19 November 1984, in final form 11 January 1985

\begin{abstract}
The VUV line emission of a high-current DC hollow cathode was investigated in the wavelength region $10 \mathrm{~nm}$ to $100 \mathrm{~nm}$. Spectra of quadruply ionised atoms could be observed. The radiance in the Al IV lines at $13 \mathrm{~nm}$ and $16 \mathrm{~nm}$ and in the $\mathrm{He}$ II Lyman-series was determined by a comparison with the spectral concentration of radiant intensity of the synchrotron radiation emitted by the electron storage ring BESSY. We found the radiance of the lines to be reproducible within $\pm 25 \%$.
\end{abstract}

\section{Introduction}

In the last decade the use of VUV radiation has steadily been growing. Today radiometric techniques are well established with calibrated transfer standard sources for wavelength $\geqslant 115 \mathrm{~nm}$, such as the deuterium lamp (Einfeld et al 1978, Key and Preston 1980), and the wall-stabilised argon mini-arc (Bridges and Ott 1977, Einfeld et al 1979). In the wavelength region below $115 \mathrm{~nm}$, reliable and easy to use transfer standard sources are still missing. It seems possible that laser-produced plasmas can be used for this purpose (Kühne 1982), but as these are pulsed sources, there still will be a demand for a continuous source. Of special interest are line radiation sources which close the gap between the long wavelength limit of soft $\mathrm{x}$-ray sources of approximately $10 \mathrm{~nm}$ and the comparatively easy to produce He I resonance line at $58.4 \mathrm{~nm}$ (Paresce et al 1971. Warden and Moos 1977).

Hollow cathode discharges are known to produce significant densities of highly excited atoms and ions (Falcone and Pedrotti 1981). We have therefore investigated the VUV emission of a high-current DC hollow cathode in the region between $10 \mathrm{~nm}$ and $100 \mathrm{~nm}$. In this paper we report measurements of the line-integrated radiance of selected Al IV, He II, and He I lines performed as a radiometric comparison with the electron storage ring BESSY as a primary standard source.

\section{Hollow cathode discharge}

We used a modified version of the hollow cathode as described by Danzmann and Kock (1980). The construction is shown schematically in figure 1. It consists of two cylindrical anodes symmetrically opposing one cathode, which contains an exchangeable hollow 


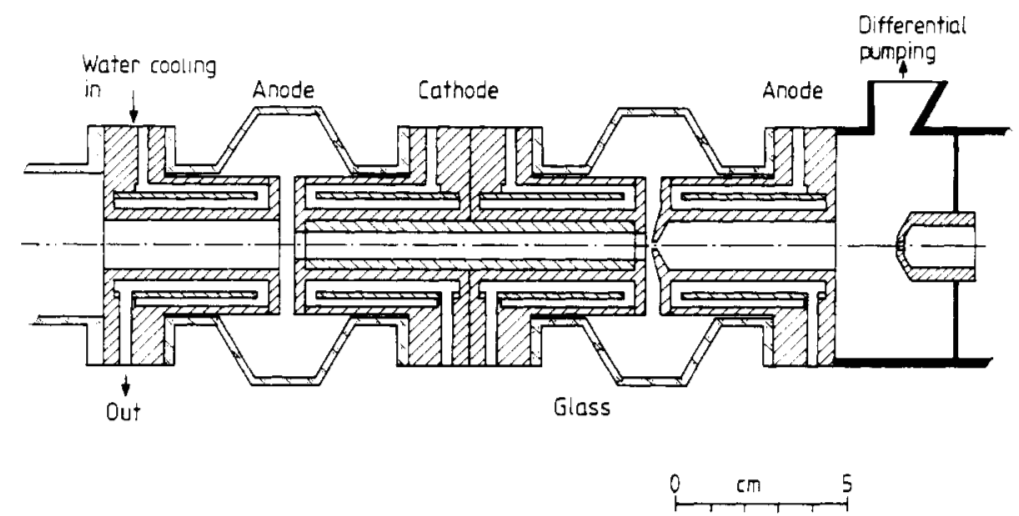

Figure 1. Longitudinal section of the hollow cathode and the differential pumping system.

metal cylinder with a straight bore of $8 \mathrm{~mm}$ diameter and $100 \mathrm{~mm}$ length. The electrical insulation of the water-cooled stainless steel electrodes is by glass tubes. The components are held together by $\mathrm{O}$-ring seals and clamps.

Reabsorption of the VUV radiation in cool boundary layers is prevented by a twostage differential pumping system. One of the anodes holds an exchangeable aperture with a free diameter of $1 \mathrm{~mm}$ at a distance of $4 \mathrm{~mm}$ from the cathode. While the

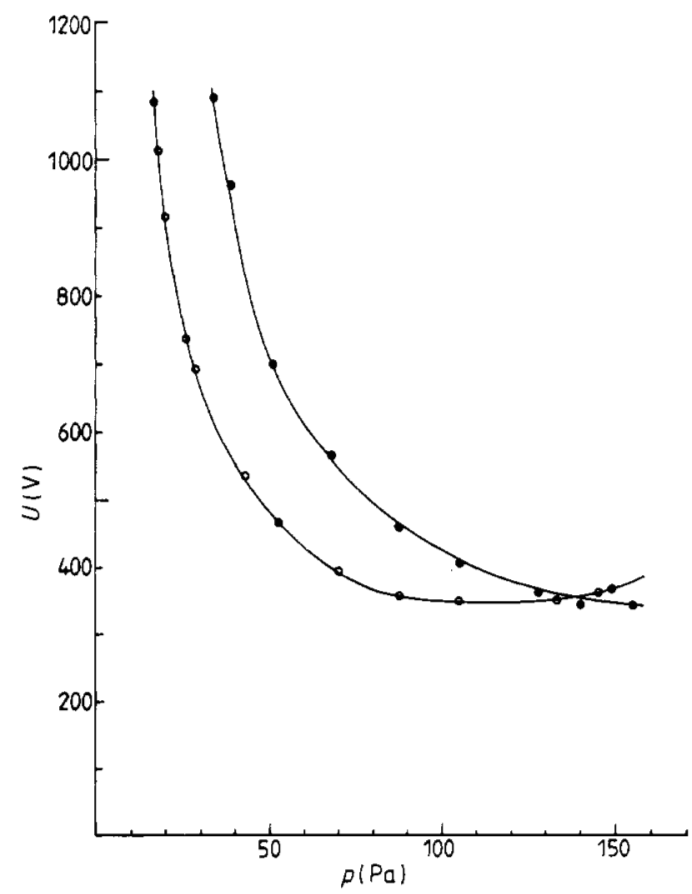

Figure 2. Operating voltage as a function of buffer gas pressure for an aluminium-argon hollow cathode. Parameter is the discharge current $I(\mathrm{~A}): 0,1 ; 0,2$. 
pressure in the discharge is typically between $10 \mathrm{~Pa}$ and $300 \mathrm{~Pa}(0.1 \mathrm{mbar}$ and $3 \mathrm{mbar})$. a turbomolecular pump evacuates the first stage to a pressure below $5 \times 10^{-2} \mathrm{~Pa}$ $\left(5 \times 10^{-4} \mathrm{mbar}\right)$. The first aperture protrudes into the negative glow region and prevents the formation of dense boundary layers. The second aperture at a distance of $100 \mathrm{~mm}$ from the first has a free diameter of $2 \mathrm{~mm}$. Behind this aperture the pressure is kept at less than $2 \times 10^{-4} \mathrm{~Pa}\left(2 \times 10^{-6} \mathrm{mbar}\right)$. We operated the discharge with cathode cylinders made out of iron, copper, vanadium, or aluminium in helium, neon. or argon as buffer gases. The voltage drop across the electrodes at constant current is dependent on the buffer gas pressure. Lower pressures require higher voltages, as can be seen from figure 2. We used a current-regulated power supply $(1500 \mathrm{~V} / 50 \mathrm{~A})$ with an RMs ripple $\Delta \mathrm{I} / \mathrm{I}=$ $10^{-3}$. The discharge voltage was adjusted by varying the buffer gas pressure. Both anodes were at earth potential. At cathode voltages larger than $1200 \mathrm{~V}$ sudden current disruptions would extinguish the discharge. Arcing limited the maximum stable currents to about $10-15$ A with new electrodes.

\section{Experimental set-up}

The experiment was performed at the laboratory for VUV radiometry of PTB at the electron storage ring BESSY. The radiances of the emission lines emitted by the discharge in the hollow cathode were determined by comparison with the calculable spectral concentration of radiant intensity of an electron storage ring (Schwinger 1949). An ellipsoidal mirror images the tangent point of the storage ring or the hollow cathode into a toroidal grating monochromator in such a way that the radiant flux is not limited by the entrance slit size (figure 3 ). For both the sources the same surface element of the mirror, the same angle of incidence and a common aperture stop $A$ is used. To allow for corrections due to the different degree of polarisation of the two sources (synchrotron radiation is highly polarised near the electron orbit plane), the monochromator can be rotated around its optical axis defined by the centre of the entrance slit and the centre of the grating. A detailed description of the calibration procedure can be found in Fischer et al (1984). The spectral concentration of radiant intensity of the hollow cathode is then converted into radiance by integrating over the line profile and averaging over the source size (aperture with $1 \mathrm{~mm}$ diameter).

An angle of incidence of $86^{\circ}$ was chosen for the ellipsoidal mirror. The distance from the storage ring tangent point to the mirror was $15000 \mathrm{~mm}$, and the distance from the $1 \mathrm{~mm}$ aperture in the differential pumping system of the hollow cathode to the mirror was $5000 \mathrm{~mm}$. The corresponding images were formed in $930 \mathrm{~mm}$ and $1060 \mathrm{~mm}$ respectively.

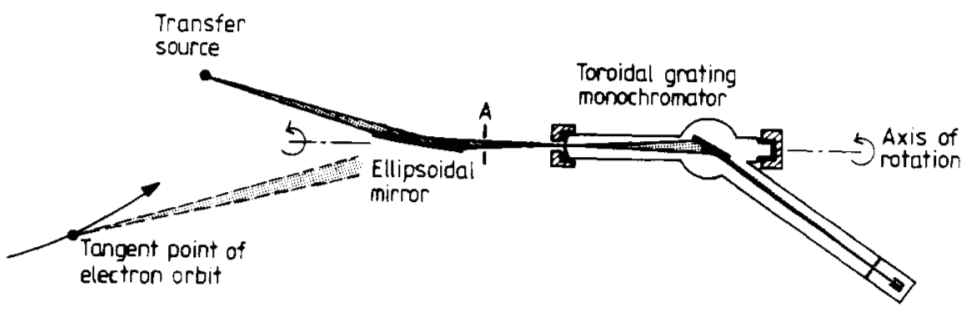

Figure 3. Instrumentation for comparing the unknown spectral concentration of radiant intensity of a source with the calculable spectral concentration of radiant intensity of an electron storage ring. 
The distance between the monochromator and the mirror could be adjusted to compensate for the different image distances. Gratings with 200 lines $/ \mathrm{mm}$ and $600 \mathrm{lines} / \mathrm{mm}$ were used with corresponding exit slit widths of $40 \mu \mathrm{m}$ and $200 \mu \mathrm{m}$ respectively to insure a bandwidth of $<0.1 \mathrm{~nm}$ for all wavelengths. The vuV signal was detected by a fast linear focused EMI D233B electron multiplier.

\section{Results and discussion}

Depending on the combination of cathode material and buffer gas pressure the whole range from $13 \mathrm{~nm}$ to $100 \mathrm{~nm}$ can be covered by intense line radiation. We observed lines from ionised noble gas atoms in ionisation stages up to $\mathrm{Ne} I \mathrm{~V}$ and $\mathrm{Ar} \mathrm{V}$. When operated at high current and low pressure, cathode material will be sputtered off the cathode wall and lines from metal ions up to $\mathrm{Al} \mathrm{V}$ and $\mathrm{Cu} \mathrm{V}$ are visible. The simplest spectrum is provided by an aluminium hollow cathode operated in helium. It mainly consists of the intense A1 IV doublet ( $\left.2 p^{6}-2 p^{5} 3 s\right)$ at $16 \mathrm{~nm}$, the He II Lyman series and He I lines. If argon is used as a buffer gas an additional Al IV line $\left(2 p^{6}-2 p^{5} 3 d\right)$ appears at $13 \mathrm{~nm}$ as can be seen in figure 4 . The cluster of lines at $17 \mathrm{~nm}$ consists of the Al III satellite lines

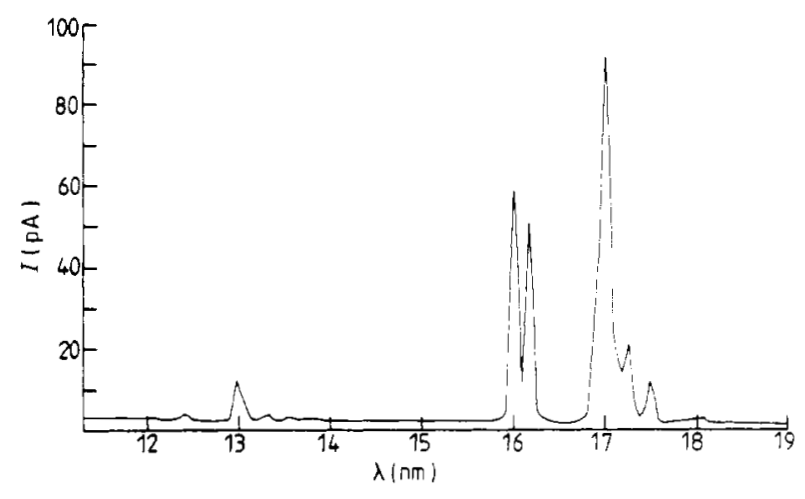

Figure 4. VUv spectrum of an aluminium-argon hollow cathode at a current of $4 \mathrm{~A}$ and a voltage of $600 \mathrm{~V}$. The photocurrent is not corrected for the spectral sensitivity of our detection system

to the Al IV lines at $16 \mathrm{~nm}$. The upper levels of these lines arise from the doubly excited configurations $2 \mathrm{p}^{5} 3 \mathrm{~s} 31$ in sodium-like Al III. Harris et al (1984) have recently noted the existence of a subclass of quartet levels of these configurations in alkali-like atoms and ions that retain relative metastability against auto-ionisation and are radiatively allowed. They discuss these states as possible upper levels of XUV lasers. It may be interesting to mention that in our DC discharge the intensity of the satellite lines is greater than that of the parent lines. The structure of the satellite cluster is dependent on the buffer gas used. If we operate our hollow cathode in neon at least nine lines are clearly visible, whereas only three lines can be distinguished when helium or argon are used. These satellite lines have been observed by Warden and Moos (1977) in a Penning discharge, but in their case the satellites were much weaker than the parent lines.

Figure 5 shows the radiance of the Al IV doublet at $16 \mathrm{~nm}$ as a function of the voltage drop across the electrodes at constant current for a $\mathrm{He}-\mathrm{Al}$ discharge. The 


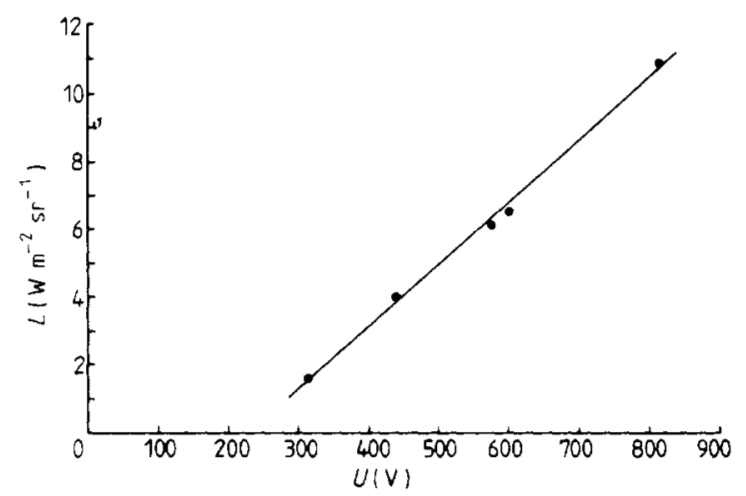

Figure 5. Radiant power of the Al IV doublet $(16.0 \mathrm{~nm}, 16.2 \mathrm{~nm})$ as a function of the discharge voltage for an aluminium-helium hollow cathode at a current of $4 \mathrm{~A}$.

voltage drop was varied by changing the buffer gas pressure. The approximately linear dependency holds for all lines in the parameter range investigated, except for neutral transitions, the radiances of which tend to saturate above $600 \mathrm{~V}$. Figure 6 shows the radiance of the Al IV doublet as a function of discharge current at constant voltage for a $\mathrm{He}-\mathrm{Al}$ discharge. The approximately quadratic dependency holds only for metal lines: noble gas lines show an almost linear behaviour. The emitted radiances are stable to within $5 \%$ for several hours, but tend to decrease because the aperture protruding into the negative glow plasma gets clogged by a deposit of sputtered cathode material. After approximately 10 h of high current operation the radiances have typically fallen to about $50 \%$ of their initial value. Removing the deposit will restore the original radiance to within our uncertainty limits. After approximately $20 \mathrm{~h}$ of high-current operation the discharge tends to become critically unstable because the cathode cylinder is degrading by ion bombardment. Replacing the cathode cylinder by a new one will restore the original performance.

We found current and voltage drop to determine the discharge in a much more meaningful way than current and buffer gas pressure, because in the low pressure regime a very small pressure change corresponds to a large change in discharge voltage. Especially with slightly degraded cathodes we sometimes observed sudden changes in

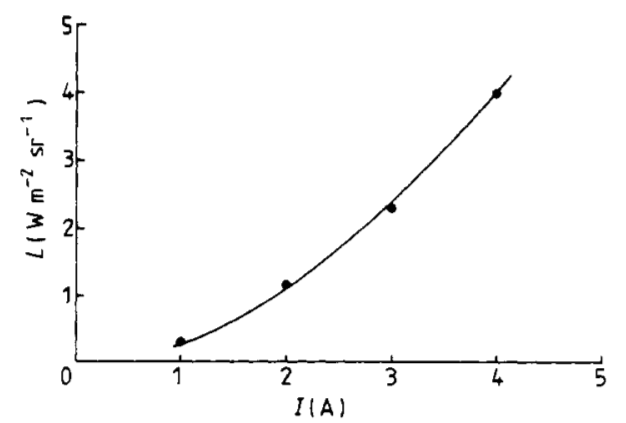

Figure 6. Radiant power of the Al IV doublet $(16.0 \mathrm{~nm}, 16.2 \mathrm{~nm})$ as a function of the discharge current for an aluminium-helium hollow cathode at a voltage of $440 \mathrm{~V}$. 
the line radiances and the voltage drop without any measureable pressure change. Adjusting the gas flow to restore the previous voltage would also restore the previous radiances.

Operating the hollow cathode at a current of $4 \mathrm{~A}$ and a voltage of $600 \mathrm{~V}$ proved to be a good compromise between high radiance and long lifetime. Under these conditions we have determined the radiance integrated over the respective line profiles for selected transitions emitted by an aluminium hollow cathode with helium or argon as buffer gases (table 1). The quoted uncertainty is the statistical uncertainty (standard deviation)

Table 1. Radiance of selected spectral lines.

\begin{tabular}{|c|c|c|c|c|}
\hline \multicolumn{3}{|c|}{$\begin{array}{l}\text { Source: } \\
\text { Cathode: } \\
\text { Buffer gases: } \\
\text { Parameters: } \\
\text { Solid angle of observation: } \\
\text { Area of observation: }\end{array}$} & \multicolumn{2}{|c|}{$\begin{array}{l}\text { Differentially pumped hollow cathode } \\
\text { Aluminium, length } 100 \mathrm{~mm} \text {, linear diameter } 8 \mathrm{~mm} \\
\text { Helium, argon } \\
I=4 \mathrm{~A}, U=600 \mathrm{~V} \\
10^{-6} \mathrm{sr} \\
\text { Circular with } 1 \mathrm{~mm} \text { diameter }\end{array}$} \\
\hline$\dot{\lambda}(\mathrm{nm})$ & Ion & Buffer gas & $L\left(\mathrm{~W} \mathrm{~m}^{-2} \mathrm{sr}^{-1}\right)$ & Uncertainty \\
\hline 13.0 & $\mathrm{Al} \mathrm{IV}$ & Argon & 0.87 & $\pm 25 \%$ \\
\hline $16.0 / 16.2$ & $\mathrm{Al} \mathrm{IV}$ & Argon & 8.2 & $\pm 25 \%$ \\
\hline $16.0 / 16.2$ & Al IV & Helium & 6.5 & $\pm 25 \%$ \\
\hline 23.7 & He II & Helium & 33 & $\pm 20 \%$ \\
\hline 24.3 & He II & Helium & 93 & $\pm 20 \%$ \\
\hline 25.6 & He II & Helium & 270 & $\pm 20 \%$ \\
\hline 30.4 & He II & Helium & 1300 & $\pm 20 \%$ \\
\hline 58.4 & $\mathrm{He} \mathrm{I}$ & Helium & 2300 & $\pm 20 \%$ \\
\hline
\end{tabular}

evaluated from five hollow cathode runs combined with three synchrotron runs. For possible systematic uncertainties see Fischer et al (1984).

It might be interesting to note that the radiant intensity obtained in the $\mathrm{He}$ II $\mathrm{Ly}-\alpha$ line from the hollow cathode is only three orders of magnitude less than the spectral concentration of radiant intensity of the BESSY storage ring ( $765 \mathrm{MeV}, 100 \mathrm{~mA}$ ) integrated over the $0.1 \mathrm{~nm}$ bandwidth of our monochromator at the same wavelength.

\section{Conclusion}

A differentially pumped high-current hollow cathode may be used as a source of intense line radiation in the VUV down to $13 \mathrm{~nm}$. Stability and reproducibility are not yet completely satisfactory for its use as a transfer standard, but it may be appropriate for general survey work not requiring high precision radiance data.

\section{Acknowledgments}

We gratefully acknowledge fruitful discussions with B Wende and $\mathrm{K}$ Grützmacher. We are indebted to $\mathrm{M} \mathrm{Kock}$, Institut für Plasmaphysik, Hannover, who provided the hollow cathode. 


\section{References}

Bridges J M and Ott W R 1977 Appl. Opt. 16 367-76

Danzmann K and Kock M 1980 J. Phys. B: At. Mol. Phys. 13 2051-59

Einfeld D, Grützmacher K and Stuck D 1979 Z. Naturf. 34a 233-38

Einfeld D, Stuck D and Wende B 1978 Metrologia 14 111-22

Falcone R W and Pedrotti 1981 Opt. Lett. 774-6

Fischer J, Kühne M and Wende B 1984 Appl. Opt. 23 4252-60

Harris S E, Walker D J, Caro R G, Mendelsohn A J, Cowan R D 1984 Opt. Lett. 9 168-70

Key P J and Preston R C 1980 J. Phys. E: Sci. Instrum. 13 866-70

Kühne M 1982 Appl. Opt. $212124-28$

Paresce F, Kumar S and Bowyer C S 1971 Appl. Opt. $101904-8$

Schwinger J 1949 Phys. Rev. 75 1912-25

Warden E S and Moos H W 1977 Appl. Opt. 16 1902-4 